\section{(1) \\ CrossMark}

\title{
Treatment of progressive fibrosing interstitial lung diseases: a milestone in the management of interstitial lung diseases
}

\author{
Vincent Cottin (1) \\ Affiliation: National Reference Centre for Rare Pulmonary Diseases, Louis Pradel Hospital, Hospices Civils de \\ Lyon, UMR754, Claude Bernard University Lyon 1, Lyon, France. \\ Correspondence: Vincent Cottin. National Reference Centre for Rare Pulmonary Diseases, Louis Pradel \\ Hospital, Hospices Civils de Lyon, UMR754, Claude Bernard University Lyon 1, Lyon, France. \\ E-mail: vincent.cottindachu-lyon.fr
}

@ERSpublications

Fibrosing ILDs can develop a progressive phenotype and are described under the terminology PFILDs. Due to commonalities with IPF, the potential efficacy and tolerability of antifibrotic drugs pirfenidone and nintedanib are currently evaluated in PF-ILDs. http://bit.ly/2koL0A4

Cite this article as: Cottin V. Treatment of progressive fibrosing interstitial lung diseases: a milestone in the management of interstitial lung diseases. Eur Respir Rev 2019; 28: 190109 [https://doi.org/10.1183/ 16000617.0109-2019].

Interstitial lung disease (ILD) refers to a large and heterogeneous group of parenchymal lung disorders [1], some related to other diseases such as connective tissue diseases (CTDs), some related to environmental exposures such as hypersensitivity pneumonitis, and others with an unknown cause and no identified aetiologic context. Idiopathic pulmonary fibrosis (IPF) is the most common type of idiopathic ILD, the most severe of the chronic forms of ILDs and represents, by definition, the prototype of progressive fibrosing ILD characterised by decline in lung function and early mortality [2].

In addition to IPF, a number of fibrosing ILDs can develop a progressive phenotype characterised histologically by self-sustaining fibrosis, a process common to a variety of conditions, and which leads to worsening quality of life, decline in lung function and, eventually, early mortality. Because these conditions share similarities regarding pathogenesis and clinical behaviour, they are increasingly described under the umbrella terminology of "progressive fibrosing ILDs" (PF-ILDs) or "fibrosing ILD with a progressive phenotype" $[3,4]$. In this editorial, PF-ILDs will refer to fibrosing ILDs other than IPF which have a progressive phenotype.

Importantly, grouping PF-ILDs creates a novel opportunity for clinical research and possibly for treatment. Indeed, no drugs are approved at present for the treatment of fibrotic ILDs other than nintedanib and pirfenidone for the treatment of IPF. Many patients with PF-ILDs are currently orphan of evidence-based treatment, and often receive corticosteroids and/or off-label immunosuppressive therapy with a variable outcome. Because of commonalities between IPF and PF-ILDs, it has been proposed that the potential efficacy and tolerability of the antifibrotic drugs pirfenidone and nintedanib be evaluated in PF-ILDs.

In this issue of the European Respiratory Review, Collins and Raghu, a pioneer and leader in the field, comprehensively review the current knowledge and ongoing research of antifibrotic therapy for PF-ILDs

Provenance: Commissioned article, peer reviewed.

Received: 19 Aug 2019 | Accepted: 13 Sept 2019

Copyright $\odot$ ERS 2019. This article is open access and distributed under the terms of the Creative Commons Attribution Non-Commercial Licence 4.0. 
beyond IPF [5]. They should be commended for such an insightful, state-of-the-art review. The concept of PF-ILDs was developed in a recent issue of the European Respiratory Review [6-13].

\section{What are the PF-ILDs?}

Aside from IPF, certain other types of chronic fibrosing ILD are also at risk of developing a progressive phenotype (figure 1) [6], and mostly include idiopathic nonspecific interstitial pneumonia, unclassifiable ILD, autoimmune ILDs especially rheumatoid arthritis-associated ILD, chronic hypersensitivity pneumonitis, genetic pulmonary fibrosis, and exposure-related diseases.

It is important to stress that only a proportion of the conditions listed above may develop a progressive phenotype. Although potentially within the spectrum of PF-ILDs, sarcoidosis (even chronic) and some of the non-IPF idiopathic interstitial pneumonias (e.g. cryptogenic organising pneumonia, desquamative interstitial pneumonia and respiratory bronchitis-ILD) rarely have a relentless, progressive course with worsening fibrosis despite appropriate therapy.

Although the exact proportion is unknown, it has been estimated based on a survey and insurance claims in the USA that $18-32 \%$ of patients diagnosed with non-IPF ILDs would develop progressive fibrosis [14]. In the same study, time from symptom onset to death was estimated to be 61-80 months [14], a poor survival yet better than that of IPF. The incidence and prevalence of PF-ILDs are not well defined [10], partly due to the heterogeneous nature of this group, and further research is clearly warranted in this area.

\section{Defining progression in patients with fibrosing ILD}

Now that the spectrum of PF-ILDs has been delineated (corresponding to fibrosing ILDs with a progressive phenotype), the next decisive question is how the term "progressive" should be defined.

First, it should be stressed that the terminology of "progressive phenotype" implies that progression of disease has occurred despite state-of-the-art management, including in many cases the use of corticosteroids and/or immunosuppressive therapy. Indeed, most of the conditions comprised within PF-ILDs, especially idiopathic nonspecific interstitial pneumonia, chronic fibrosing hypersensitivity pneumonitis, CTD-associated ILDs and unclassifiable fibrotic ILD, histologically consist of a variable proportion and combination of inflammation and fibrosis. Therefore, therapies targeting inflammation will be effective at least to some extent in most patients and should represent the mainstay of treatment. It is

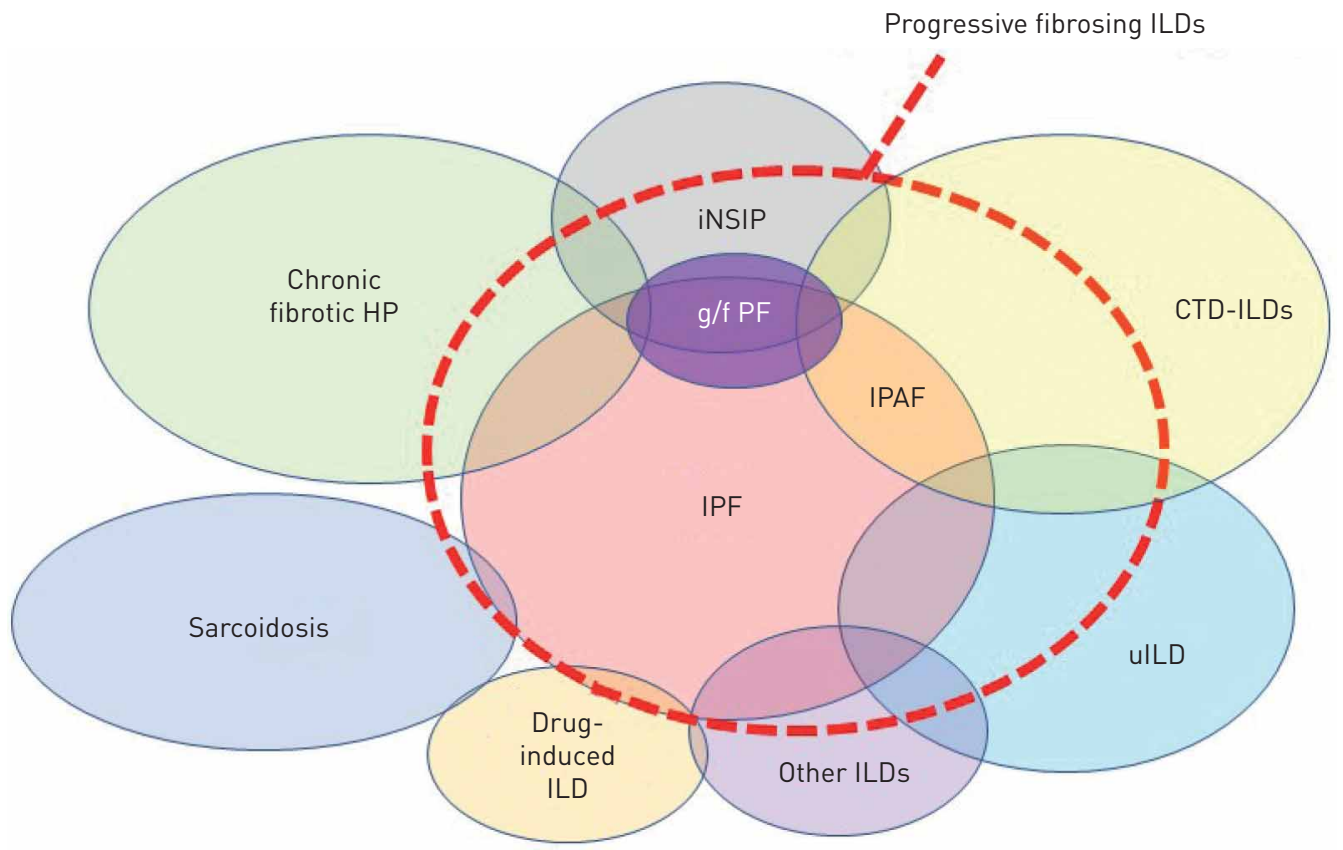

FIGURE 1 Schematic representation of types of interstitial lung disease (ILD) that may be associated with a progressive fibrosing phenotype. Connective tissue disease (CTD)-ILDs include rheumatoid arthritis-associated ILD, systemic sclerosis-associated ILD, mixed CTD-associated ILD and other autoimmune ILDs. Other ILDs include exposure-related ILDs (asbestosis and silicosis), non-idiopathic pulmonary fibrosis (IPF) idiopathic interstitial pneumonias (desquamative interstitial pneumonia, etc.), and others. g/f PF: genetic and/or familial pulmonary fibrosis; HP: hypersensitivity pneumonitis; iNSIP: idiopathic nonspecific interstitial pneumonia; IPAF: interstitial pneumonia with autoimmune features; uILD: unclassifiable ILD. 


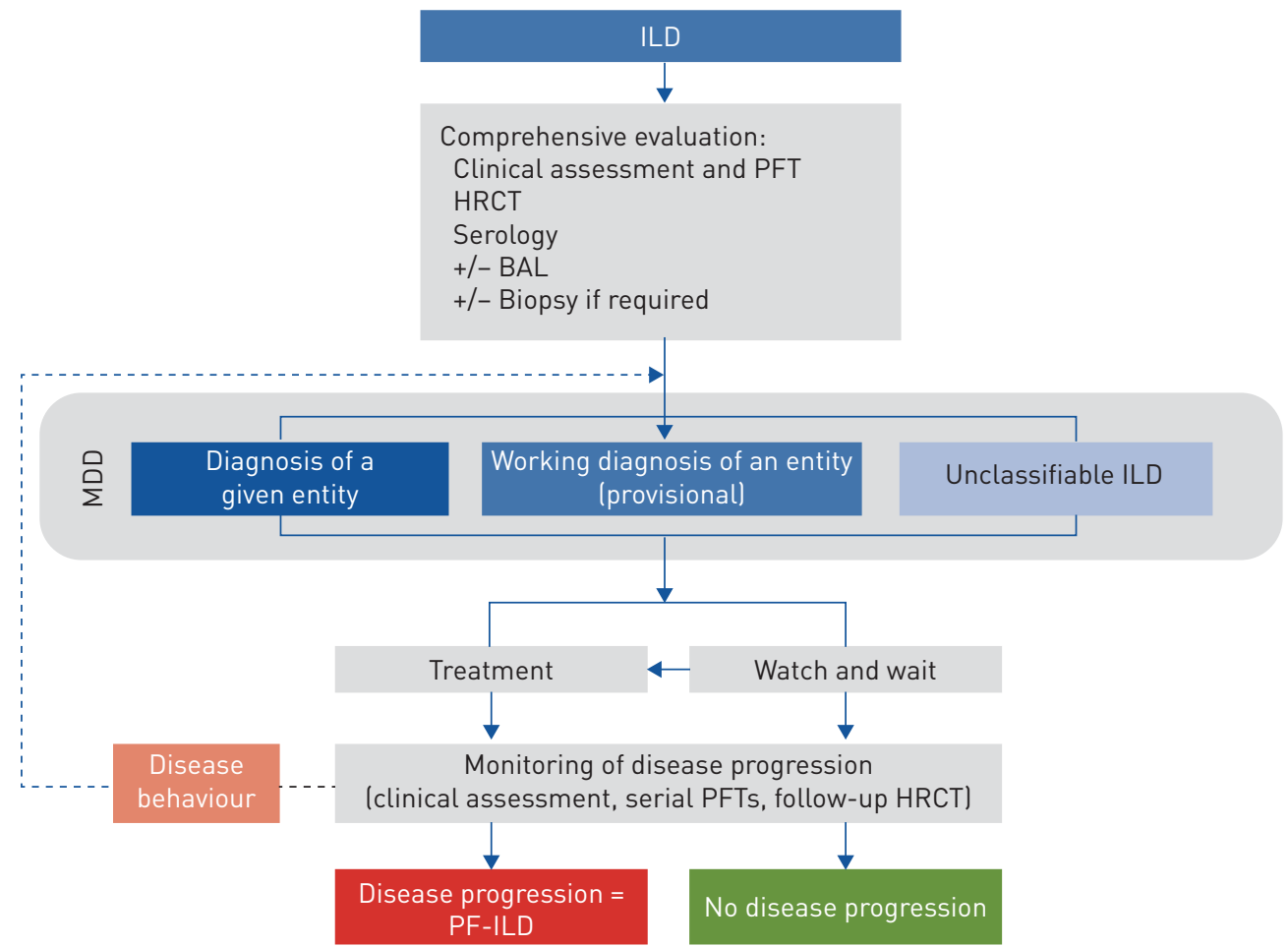

FIGURE 2 Diagnosis of fibrosing interstitial lung diseases (ILDs) that may present a progressive phenotype. BAL: bronchoalveolar lavage; HRCT: high-resolution computed tomography; MDD: multidisciplinary diagnosis; PF-ILD: progressive-fibrosing ILD; PFT: pulmonary function test. Reproduced from [6].

only when fibrosis progresses despite corticosteroids and/or immunosuppressive therapy, sometimes including several lines of different drugs, that a progressive phenotype may be considered (figure 2), and that the need for alternative therapy may be contemplated.

There is no consensus as to how disease progression should be defined in patients with ILDs, and a number of end-points have been proposed in clinical trials of fibrosing ILDs [6]. In IPF, most studies have defined disease progression in terms of a decline in forced vital capacity (FVC), measured as the change from baseline or as a categorical change (typically $\geqslant 10 \%$ predicted), or more recently as a composite of categorical change and mortality [15]. Decline in FVC is a well-established predictor of mortality in IPF [16]; however, in clinical practice no threshold or rate of decline has been formally accepted to assess progression. Patient-reported outcomes, imaging features, acute worsening events, mortality, exercise capacity and health-related quality of life measures are often used as secondary end-points.

In clinical practice, however, monitoring of fibrosing ILDs usually includes multiple components, consisting of symptoms and exercise capacity, decline in FVC and diffusing capacity of the lungs for carbon monoxide $\left(D_{\mathrm{LCO}}\right)$ and, less frequently, worsening of fibrotic features at serial high-resolution computed tomography (HRCT) (table 1). Deterioration in health-related quality of life is highly meaningful for the patients but lacks objectivity in practice. Non-elective hospitalisation [16], acute worsening of shortness of breath and initiation of ambulatory or long-term supplemental oxygen [18] are directly relevant to the patients, predict long-term mortality in IPF [19], and may be used as landmarks to assess progression and the effects of treatment interventions throughout the course of disease.

Overall, change in FVC represents the predominant tool to assess disease progression, and may be supported by changes on HRCT or in symptoms, especially when change in lung function is marginal and may be confounded by measurement variability. Monitoring of FVC can also be confounded by comorbid emphysema [20]. In the absence of a uniformly accepted definition, it has been suggested [3, 4, 17] that patients with an absolute fall of $\geqslant 5 \%$ in FVC, or a fall of $\geqslant 15 \%$ in $D_{\text {LCO }}$ [21], or worsening symptoms or worsening radiological appearance accompanied by a marginal decrease in FVC or $D_{\text {LCO }}$ within a 24-month period have experienced disease progression [3]. Comparable criteria were used in the INBUILD trial to assess disease progression and eligibility based on disease course prior to enrolment in the trial (table 2) [3].

It should be kept in mind that the course of disease for an individual patient remains impossible to predict. Previous decline in FVC is not a good predictor of future decline in FVC [24, 25]. Lower FVC, 
TABLE 1 Proposed criteria that may be used in clinical practice to assess disease progression in fibrotic interstitial lung diseases

Lung function

Exercise capacity

\section{Symptoms and patient-reported outcomes}

Acute worsening

HRCT

Need for supportive care

Serum biomarkers
Rate of decline in FVC $\left(\mathrm{mL} \cdot\right.$ year $\left.^{-1}\right)$

Absolute or relative changes in FVC ( $\mathrm{mL}$ or \% predicted) Absolute or relative changes in $D_{\mathrm{LCO}} \%$ predicted Absolute change in 6-min walk test distance Change in oxygen saturation nadir during 6-min walk test Change in maximal exercise capacity Change in symptoms

Change in everyday life exercise capacity

Questionnaires on shortness of breath, cough, and/or quality of life

Acute exacerbation of fibrosis (idiopathic or triggered)

Non-elective hospitalisation for a respiratory cause

Change in the extent or texture of fibrotic features on HRCT

Change in quantitative fibrosis scores on $\mathrm{HRCT}^{\#}$

Initiation of ambulatory oxygen therapy at exercise

Initiation of supplemental oxygen therapy at rest, or change in

flow of oxygen

None validated

Not yet applicable in clinical practice

As these criteria are intended to guide individual decisions in clinical practice, they may differ from end-points used in clinical trials [17]. Most clinicians would make management decisions based on a combination of variables. HRCT: high-resolution computed tomography; FVC: forced vital capacity; $D_{\text {Lco: }}$ diffusing capacity of the lung for carbon monoxide. " : not yet routinely available.

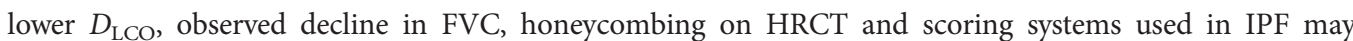
predict disease progression in PF-ILDs [26], however, further study is needed. In the future, the use of biomarkers, none of them are validated in fibrotic ILDs, may make the concept of precision medicine a reality in PF-ILDs. There is research to identify patient subgroups based on genetic or molecular profiles, or on environmental or behavioural factors, to enable better prediction of the course of disease for individual patients and facilitate the selection of the most appropriate therapies.

\section{Antifibrotic therapy could be beneficial in PF-ILDs}

Because PF-ILDs have been defined by progression despite conventional therapy, there is inherently a huge unmet need for the treatment of these conditions. Antifibrotic drugs effective to slow down disease progression in IPF may conceivably have a comparable efficacy in other PF-ILDs. It is postulated that once the response to lung injury in fibrosing ILDs has reached the stage at which fibrosis has become progressive and self-sustaining, targeted anti-fibrotic therapy would be required to slow disease progression.

Support of this concept has emanated from in vitro studies and animal models for both nintedanib and pirfenidone. Nintedanib, an intracellular inhibitor of tyrosine kinases [27, 28], has shown antifibrotic, anti-inflammatory and vascular remodelling effects in several non-clinical models of fibrosis [29, 30]. Pirfenidone, a small molecule with antifibrotic, anti-inflammatory and anti-oxidative effects, suppresses the production of transforming growth factor- $\beta$, the proliferation and differentiation of myofibroblasts, and the deposition of collagen, and has favourable effects in non-clinical models of lung fibrosis [31-34]. Both drugs have demonstrated efficacy in IPF and could be effective to slow down progressive fibrosis in PF-ILDs irrespective of the trigger for the injury. Conceivably, these drugs might also be beneficial when fibrosis affects organs other than the lungs, as fibrogenesis is a very general process in the body [35].

As comprehensively reviewed by Collins and RAGHU [5], a number of clinical trials are on-going to assess the efficacy and tolerability of antifibrotics in a number of conditions. Some of the trials enrol patients with one of the conditions belonging to PF-ILDs, for example rheumatoid arthritis-associated ILD (identifier NCT02808871; www.clinicaltrials.gov), systemic sclerosis-associated ILD [36] or unclassifiable ILD [22], whereas others were designed to enrol a large group of patients fulfilling predefined criteria for all PF-ILDs $[3,23]$. Given the rarity of most of the conditions composing PF-ILDs, "basket" trials that enrol various conditions are a unique opportunity for research. If the trials are positive, it is anticipated that the homogeneity of the drug effect will be assessed across different disease subgroups.

The authors of the review are hopeful that antifibrotic drugs may be beneficial in PF-ILDs [5], based on convincing biological evidence, data from animal models, and the fact that both available antifibrotic 
TABLE 2 Definition of disease progression in the main ongoing studies involving patients with progressive fibrosing interstitial lung diseases

\begin{tabular}{|c|c|c|c|}
\hline $\begin{array}{l}\text { Study name and } \\
\text { registration number }\end{array}$ & $\begin{array}{l}\text { Period considered to } \\
\text { assess progression }\end{array}$ & Criteria & [Ref.] \\
\hline $\begin{array}{l}\text { INBUILD, } \\
\text { NCT02999178 }\end{array}$ & $\begin{array}{l}24 \text { months prior to } \\
\text { screening }\end{array}$ & $\begin{array}{c}\text { Relative decline in FVC } \geqslant 10 \% \text { predicted } \\
\text { Relative decline in FVC } \geqslant 5-<10 \% \text { predicted } \\
\text { combined with increased extent of fibrosis on } \\
\text { HRCT } \\
\text { Relative decline in FVC } \geqslant 5-<10 \% \text { predicted } \\
\text { combined with worsening of respiratory } \\
\text { symptoms } \\
\text { Worsened respiratory symptoms and increased } \\
\text { extent of fibrosis on HRCT only }\end{array}$ & [3] \\
\hline ulLD, NCT03099187 & $\begin{array}{l}6 \text { months prior to } \\
\text { screening }\end{array}$ & $\begin{array}{l}>5 \% \text { absolute decline in FVC \% predicted } \\
\text { Significant symptomatic worsening not due to } \\
\text { cardiac, pulmonary lexcept worsening of } \\
\text { underlying ulLD), vascular or other causes las } \\
\text { determined by the investigator) }\end{array}$ & [22] \\
\hline $\begin{array}{l}\text { RELIEF, } \\
\text { DRKS00009822 }\end{array}$ & $\begin{array}{l}6 \text { months prior to } \\
\text { inclusion }\end{array}$ & $\begin{array}{l}\text { Slope calculation of at least three values } \\
\text { documenting an annualised decline in FVC \% } \\
\text { predicted of } 5 \% \text { (absolute) or more despite } \\
\text { appropriate conventional therapy }\end{array}$ & [23] \\
\hline
\end{tabular}

One criterion was sufficient for eligibility in each of the respective studies. uILD: unclassifiable interstitial lung disease; FVC: forced vital capacity; HRCT: high-resolution computed tomography.

drugs share a broad mode of action (including some anti-inflammatory effects in vitro). They further speculate that some of the published IPF trials may have spuriously included patients with non-IPF fibrotic diseases, and suggest that the fact that these IPF trials were positive despite the fact that they included patients with non-IPF PF-ILDs, could be seen as indirect evidence that the antifibrotic drugs may also provide a benefit in such cases. Support for the potential efficacy of antifibrotic drugs in PF-ILDs has also stemmed from the recent publication of the SENSCIS trial [34], which showed a $44 \%$ relative reduction in the rate of decline in FVC over time in patients with systemic sclerosis. The results of the other trials in PF-ILDs are eagerly awaited.

\section{An expected change in paradigm}

If a benefit of antifibrotic therapy is demonstrated in PF-ILDs by ongoing trials, major changes may be expected in how fibrotic ILDs are diagnosed and managed in the future. A precise diagnosis of a given entity might become less crucial than it currently is, as management would be mostly guided by establishing a diagnosis of fibrosing ILD, assessment of relative contribution of inflammation and fibrosis to the disease process, and assessment of disease behaviour with first-line therapy. The value of a precise diagnosis in the long-term management of any patient with ILD should, however, not be underestimated. As one important example, immunosuppressive therapy is deleterious in IPF, and therefore an accurate diagnosis of IPF is mandatory.

Next, if trials of antifibrotic therapy in PF-ILDs are positive, progress and standardisation will be needed in how we assess disease progression. In other words, identification of progressive disease would become key in the disease management. In addition to assessment using serial pulmonary function tests, easily available biomarkers reliably measuring disease activity would be eagerly needed, especially to differentiate progression of fibrosis from progression of inflammation (or from symptoms caused by permanent, non-progressing defects). In the era of precision medicine, evaluation of disease progression would be one component of a multicompartment disease assessment, with the disease category being one of them.

Obviously, if the trials of PF-ILDs with antifibrotic therapy are positive, major changes are to be expected in how a vast proportion of patients suffering from these conditions would be treated, as antifibrotic therapy would no longer be considered a treatment of IPF but of progressive fibrosis whatever the aetiology. However, antifibrotic therapy would likely be restricted to those patients experiencing a progressive fibrotic phenotype despite conventional management including, in many cases, corticosteroids and/or immunosuppressive therapy. The combination of an immunomodulating treatment with an antifibrotic drug is tolerable [36, 37]. It might be beneficial in some cases [38] as suggested by some 
animal models [32] and by the study of nintedanib in patients with systemic sclerosis, about half of them were receiving concomitant mycophenolate mofetil [36].

The results of the current trials will also shape the design of future trials of antifibrotic drugs. Novel drugs in development will be evaluated in IPF, as well as in other PF-ILDs, alone and in combination, with many combinations already envisioned. Eventually, broadening the scope of conditions which might benefit from antifibrotic therapy may boost this already very active arena.

The review by Collins and RAGHU [5] opens up new perspectives in an almost virgin field. Let's prepare for a change in paradigm.

Conflict of interest: V. Cottin reports personal fees and non-financial support from Actelion, grants, personal fees and non-financial support from Boehringer Ingelheim and Roche, and personal fees from Bayer/MSD, Gilead, Novartis, Sanofi, Promedior, Celgene, Galapagos and Galecto, outside the submitted work.

\section{References}

1 Travis WD, Costabel U, Hansell DM, et al. An official American Thoracic Society/European Respiratory Society statement: update of the international multidisciplinary classification of the idiopathic interstitial pneumonias. $A m$ J Respir Crit Care Med 2013; 188: 733-748.

2 Raghu G, Collard HR, Egan JJ, et al. An Official ATS/ERS/JRS/ALAT statement: idiopathic pulmonary fibrosis: evidence-based guidelines for diagnosis and management. Am J Respir Crit Care Med 2011; 183: 788-824.

3 Flaherty KR, Brown KK, Wells AU, et al. Design of the PF-ILD trial: a double-blind, randomised, placebo-controlled phase III trial of nintedanib in patients with progressive fibrosing interstitial lung disease. $B M J$ Open Respir Res 2017; 4: e000212.

4 Wells AU, Brown KK, Flaherty KR, et al. What's in a name? That which we call IPF, by any other name would act the same. Eur Respir J 2018; 51: 1800692.

5 Collins BF, Raghu G. Antifibrotic therapy for fibrotic lung disease beyond idiopathic pulmonary fibrosis. Eur Respir Rev 2019; 28: 190021

6 Cottin V, Hirani NA, Hotchkin DL, et al. Presentation, diagnosis and clinical course of the spectrum of progressive-fibrosing interstitial lung diseases. Eur Respir Rev 2018; 27: 180076.

7 Harari S. Beyond idiopathic pulmonary fibrosis: the world of progressive-fibrosing interstitial lung disease. Eur Respir Rev 2018; 27: 180110.

8 Holtze C, Flaherty K, Kreuter M, et al. Healthcare utilisation and costs in the diagnosis and treatment of progressive-fibrosing interstitial lung diseases. Eur Respir Rev 2018; 27: 180078.

9 Kolb M, Bondue B, Pesci A, et al. Acute exacerbations of progressive-fibrosing interstitial lung diseases. Eur Respir Rev 2018; 27: 180071.

10 Olson AL, Gifford AH, Inase N, et al. The epidemiology of idiopathic pulmonary fibrosis and interstitial lung diseases at risk of a progressive-fibrosing phenotype. Eur Respir Rev 2018; 27: 180077.

11 Richeldi L, Varone F, Bergna $\mathrm{M}$, et al. Pharmacological management of progressive-fibrosing interstitial lung diseases: a review of the current evidence. Eur Respir Rev 2018; 27: 180074.

12 Swigris JJ, Brown KK, Abdulqawi R, et al. Patients' perceptions and patient-reported outcomes in progressive-fibrosing interstitial lung diseases. Eur Respir Rev 2018; 27: 180075.

13 Walsh SLF, Devaraj A, Enghelmayer JI, et al. Role of imaging in progressive-fibrosing interstitial lung diseases. Eur Respir Rev 2018; 27: 180073.

14 Wijsenbeek M, Kreuter M, Olson A, et al. Progressive fibrosing interstitial lung diseases: current practice in diagnosis and management. Curr Med Res Opin 2019: 1-10.

15 Spagnolo P, Cocconcelli E, Cottin V. Clinical trials in IPF: what are the best endpoints? In: Meyer KC, Nathan SD, eds. Idiopathic Pulmonary Fibrosis. 2nd Edn. Switzerland, Springer Nature, 2019; pp. 433-453.

16 Paterniti MO, Bi Y, Rekic D, et al. Acute exacerbation and decline in forced vital capacity are associated with increased mortality in idiopathic pulmonary fibrosis. Ann Am Thorac Soc 2017; 14: 1395-1402.

17 Hook JL, Arcasoy SM, Zemmel D, et al. Titrated oxygen requirement and prognostication in idiopathic pulmonary fibrosis. Eur Respir J 2012; 39: 359-365.

18 Sharp C, Adamali HI, Millar AB. A comparison of published multidimensional indices to predict outcome in idiopathic pulmonary fibrosis. ERJ Open Res 2017; 3: 00096-2016.

19 Cottin V, Hansell DM, Sverzellati N, et al. Effect of emphysema extent on serial lung function in patients with idiopathic pulmonary fibrosis. Am J Respir Crit Care Med 2017; 196: 1162-1171.

20 Cottin V, Wollin L, Fischer A, et al. Fibrosing interstitial lung diseases: knowns and unknowns. Eur Respir Rev 2019; 28: 180100.

21 Bradley B, Branley HM, Egan JJ, et al. Interstitial lung disease guideline: the British Thoracic Society in collaboration with the Thoracic Society of Australia and New Zealand and the Irish Thoracic Society. Thorax 2008; 63: Suppl 5, v1-58.

22 Maher TM, Corte TJ, Fischer A, et al. Pirfenidone in patients with unclassifiable progressive fibrosing interstitial lung disease: design of a double-blind, randomised, placebo-controlled phase II trial. BMJ Open Respir Res 2018; 5: e000289.

23 Behr J, Neuser P, Prasse A, et al. Exploring efficacy and safety of oral pirfenidone for progressive, non-IPF lung fibrosis (RELIEF) - a randomized, double-blind, placebo-controlled, parallel group, multi-center, phase II trial. BMC Pulm Med 2017; 17: 122.

24 Schmidt SL, Tayob N, Han MK, et al. Predicting pulmonary fibrosis disease course from past trends in pulmonary function. Chest 2014; 145: 579-585.

25 Nathan SD, Albera C, Bradford WZ, et al. Effect of continued treatment with pirfenidone following clinically meaningful declines in forced vital capacity: analysis of data from three phase 3 trials in patients with idiopathic pulmonary fibrosis. Thorax 2016; 71: 429-435.

26 Kolb M, Vasakova M. The natural history of progressive fibrosing interstitial lung diseases. Respir Res 2019; $20: 57$. 
Hilberg F, Roth GJ, Krssak M, et al. BIBF 1120: triple angio
good antitumor efficacy. Cancer Res 2008; 68: 4774-4782.
28 Hilberg F, Tontsch-Grunt U, Baum A, et al. Triple angiok

Hilberg F, Tontsch-Grunt U, Baum A, et al. Triple angiokinase inhibitor nintedanib directly inhibits tumor cell growth and induces tumor shrinkage via blocking oncogenic receptor tyrosine kinases. J Pharmacol Exp Ther 2018; 364: 494-503.

29 Wollin L, Distler JHW, Redente EF, et al. Potential of nintedanib in treatment of progressive fibrosing interstitial lung diseases. Eur Respir J 2019; 54: 1900161.

30 Redente EF, Aguilar MA, Black BP, et al. Nintedanib reduces pulmonary fibrosis in a model of rheumatoid arthritis-associated interstitial lung disease. Am J Physiol Lung Cell Mol Physiol 2018; 314: L998-L1009.

31 Schaefer CJ, Ruhrmund DW, Pan L, et al. Antifibrotic activities of pirfenidone in animal models. Eur Respir Rev 2011; 20: 85-97.

32 Rasooli R, Pourgholamhosein F, Kamali Y, et al. Combination therapy with pirfenidone plus prednisolone ameliorates paraquat-induced pulmonary fibrosis. Inflammation 2018; 41: 134-142.

33 Qin W, Liu B, Yi M, et al. Antifibrotic agent pirfenidone protects against development of radiation-induced pulmonary fibrosis in a murine model. Radiat Res 2018; 190: 396-403.

34 Guo J, Yang Z, Jia Q, et al. Pirfenidone inhibits epithelial-mesenchymal transition and pulmonary fibrosis in the rat silicosis model. Toxicol Lett 2019; 300: 59-66.

35 Rockey DC, Bell PD, Hill JA. Fibrosis - a common pathway to organ injury and failure. N Engl J Med 2015; 372: $1138-1149$.

36 Distler O, Highland KB, Gahlemann M, et al. Nintedanib for systemic sclerosis-associated interstitial lung disease. N Engl J Med 2019; 380: 2518-2528.

37 Khanna D, Albera C, Fischer A, et al. An open-label, phase ii study of the safety and tolerability of pirfenidone in patients with scleroderma-associated interstitial lung disease: the LOTUSS trial. J Rheumatol 2016; 43: 1672-1679.

38 Wuyts WA, Antoniou KM, Borensztajn K, et al. Combination therapy: the future of management for idiopathic pulmonary fibrosis? Lancet Respir Med 2014; 2: 933-942. 